\title{
Neugeborenen-Screening auf Cystische Fibrose - ab 1. Januar 2011 auch in der Schweiz
}

Jürg Barben ${ }^{a}$, Toni Torresani ${ }^{b}$, Martin H. Schönic, Sabina Gallati, Matthias Baumgartnere und task force

CFNeugeborenenScreening*

a Präsident Swiss Working Group for Cystic Fibrosis (SWGCF), Sekretär Schweizerische Gesellschaft für Pädiatrische Pneumologie (SGPP), Leitender Arzt Pneumologie/ Allergologie, Ostschweizer Kinderspital St. Gallen

b Leiter NeugeborenenScreening Schweiz und Proteinhormonlabor Endokrinologie, UniversitätsKinder-Kliniken Zürich

c Chefarzt ambulante Pädiatrie, UniversitätsKinderklinik Bern

d Leiterin Abteilung Humangenetik, Medizinische Universitäts-Kinderklinik Bern

e Leiter Abteilung Stoffwechsel und Molekulare Pädiatrie, Universitäts-Kinderkliniken Zürich

* Weitere Mitglieder: Carmen Casaulta (Bern), Anne Mornand (Genf), Ralph Fingerhut (Zürich), Gaudenz Hafen (Lausanne), Alexander Möller (Zürich), Nicolas Regamey (Bern)

Korrespondenz:

PD Dr. med. Jürg Barben Präsident SWGCF

Leitender Arzt Pneumologie/ Allergologie

Ostschweizer Kinderspital

CH-9006 St. Gallen

Tel. 0712437111

Fax 0712437390

juerg.barben@kispisg.ch
Ein Neugeborenen-Screening auf verschiedene angeborene Stoffwechsel-Krankheiten besteht in der Schweiz bereits seit über 40 Jahren und wird landläufig auch «Guthrie-Test» genannt. Dabei wird am 4. Lebenstag bei jedem Neugeborenen in der Schweiz etwas Blut an der Ferse abgenommen und auf einem Filterpapier getrocknet. Seit Herbst 2005 wird dies zentral im Neugeborenen-Screening-Labor an der Universitäts-Kinderklinik Zürich verarbeitet. Bis anhin wurde auf sechs behandelbare Krankheiten getestet; neu kommt ab 1. Januar 2011 die Cystische Fibrose hinzu (Tab. 1).

\section{Tabelle 1}

Aktuell gescreente Krankheiten im Schweizer Neugeborenen-Screening-Programm:

Phenylketonurie (PKU)

Galactosämie (Transferase, Kinase, Epimerase-Mangel)

Biotinidase-Mangel

MCADD (Medium Chain AcylCoA Dehydrogenase-Mangel)

Kongenitaler Hypothyroidismus

Kongenitales Adrenogenitales Syndrom

Neu: Cystische Fibrose (CF)

Die Cystische Fibrose (CF) ist die häufigste angeborene Stoffwechselerkrankung in der Schweiz mit einer Inzidenz von etwa 1: 2500, das heisst, die CF kommt häufiger vor als alle bisher getesteten Krankheiten. Mit Ausnahme des Mekoniumileus hat auch die CF ein symptomfreies Intervall unmittelbar nach der Geburt. Die Diagnose wurde bisher klinisch aufgrund der später auftretenden Symptome (rezidivierender Husten bzw. obstruktive Bronchitis, Gedeihstörung, Fettstühle, chronische Bauchschmerzen, chronische Rhinosinusitis bzw. Nasenpolypen usw.) mit einer Verzögerung von mehreren Monaten, teilweise auch Jahren gestellt. In den letzten Jahren haben sich die Therapiemöglichkeiten deutlich verbessert, und die Lebenserwartung hat in den letzten Jahrzehnten dramatisch zugenommen. Gemäss dem amerikanischen CF-Patientenregister beträgt heute das mediane Durchschnittsalter bereits knapp 40 Jahre und in England schätzt man das mittlere Überlebensalter auf 50 Jahre für diejenigen Patienten, die im 21. Jahrhundert geboren sind [1].

Seit 1979 gibt es mit dem immunreaktiven Trypsin (IRT) eine einfache und recht zuverlässige Analysemethode für das CF-Screening im Blut, die bei Neuge- borenen gut angewandt werden kann [2]. Die ersten grossen CF-Screening-Programme wurden bereits 1981 in Neuseeland und Australien begonnen [3]. Heute gibt es ein CF-Neugeborenen-Screening (CFNGS) in allen Staaten der USA sowie in zahlreichen europäischen Ländern wie England, Irland, Schottland, Frankreich, Österreich und Polen sowie in vielen Regionen in Italien und Spanien [4, 5]. Inzwischen gibt es auch amerikanische und europäische Richtlinien, wie solche Programme aufgebaut, umgesetzt und erfolgreich geführt werden können [6, 7].

\section{Welche Nutzen bringt ein CF-Screening?}

Bis vor 10 Jahren gab es keine kontrollierten Studien, die einen grossen Vorteil einer frühzeitigen CF-Diagnose durch ein Screening zeigten. In den letzten Jahren wurden aber zahlreiche Studien veröffentlicht, die bei frühzeitiger Diagnosestellung eine verbesserte Ernährung und besseres Wachstum, eine bessere Hirnentwicklung (infolge adäquat substituiertem Vitamin E) sowie weniger Erkrankungen bzw. Spitalaufenthalte und teilweise auch eine verbesserte Lungenfunktion zeigten $[1,8]$. Einige Studien konnten auch eine erhöhte Überlebenszeit nachweisen. Alle ScreeningProgramme weisen auf den grossen psychologischen Vorteil hin, die Leidenszeit bis zur Diagnose bzw. die Ungewissheit der Eltern zu verkürzen. Ausserdem ermöglicht die frühzeitige Diagnose eine bewusstere Familienplanung mit der Möglichkeit einer pränatalen Diagnostik bei weiterem Kinderwunsch [4].

\section{Gibt es auch Nachteile?}

Wie bei jedem Screening-Test gibt es falschpositive und falschnegative Resultate. Die falschpositiven Resultate werden mit dem bei uns eingeführten zweistufigen Verfahren (Abb. 1) möglichst klein gehalten, wobei eine optimale Balance angestrebt wurde, um möglichst viele Kinder mit CF frühzeitig zu erfassen (= gute Sensitivität), dabei aber möglichst nur diejenigen zu erfassen, die auch wirklich eine CF mit entsprechenden Symptomen entwickeln (= gute Spezifität). Der Schweisstest gibt letztendlich Aufschluss über das Vorhandensein einer CF mit relevanten Symptomen (klassische CF). Die Zeit bis zu diesem Test wird von den Eltern unterschiedlich wahrgenommen. Die falschnegativen Resultate, das heisst diejenigen, die eine CF haben, aber im Screening-Test nicht erfasst werden, werden in den USA auf ca. 2-4\% geschätzt [4]. Dies hängt hauptsächlich davon ab, nach wie vielen verschiedenen von den heute über 
1600 bekannten CF-Genmutationen beim CFNGS gesucht wird. Das bedeutet aber auch, dass auch in $\mathrm{Zu}$ kunft bei entsprechender klinischer Symptomatik immer an eine CF gedacht werden muss, denn auch das beste CFNGS kann nicht alle Kinder mit CF erfassen. Je mehr Mutationen gesucht werden, desto höher ist die Erkennungsrate von mild verlaufenden Formen von CF (atypische CF), die vielleicht erst mit 20 oder 30 Jahren Symptome entwickeln würden. Aus diesem Grunde werden im Schweizer CFNGS nur die sieben häufigsten CF-Genmutationen bestimmt. Ein weiterer möglicher Nachteil kann sein, dass gescreente Kinder mit CF schon früh in einem CF-Zentrum mit Mikroben infiziert werden können, mit denen sie nie in Kontakt gekommen wären, wenn sie nicht in ein CF-Zentrum gekommen wären. Aus diesem Grund kommt den Hygiene-Empfehlungen in den Spitälern eine grosse Bedeutung zu. Bei jedem CF-Screening werden auch gesunde Träger von CF-Mutationen gefunden (solche, die im Screening positiv sind, aber einen normalen Schweisstest haben), und es gibt Menschen, die einen solchen Trägerstatus gar nicht wissen möchten.

\section{Wie funktioniert ein CF-Screening genau?}

Wird im Guthrie-Test ein erhöhter IRT-Wert gefunden, werden im gleichen Blutstropfen die sieben in der Schweiz häufigsten Genmutationen (DANN-Analyse) gesucht. Ist auch dieser zweite Test positiv (Nach-

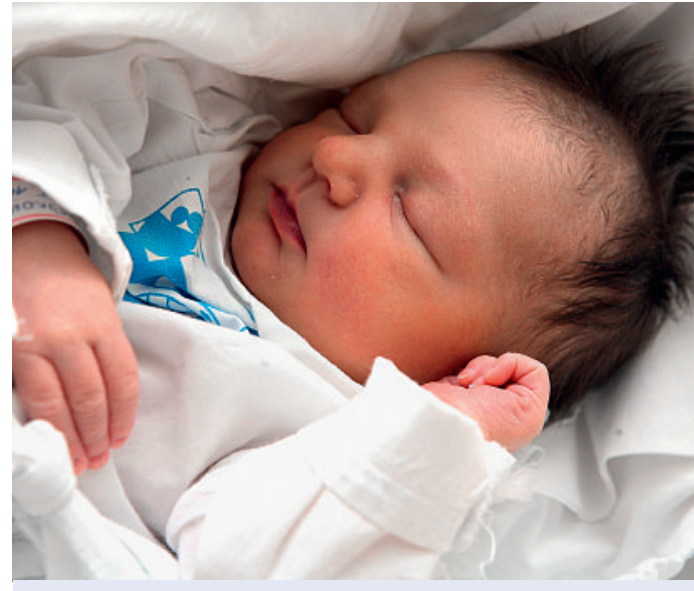

Die frühzeitige Diagnose von Cystischer Fibrose ermöglicht Verbesserungen bei Ernährung, Wachstum, Hirnentwicklung und teilweise auch bei der Lungenfunktion.

weis mindestens einer CF-Genmutation) gilt das Screening als positiv. Da das NGS eine Reihenuntersuchung gemäss dem heute geltenden Gesetz für genetische Untersuchungen beim Menschen darstellt, ist keine schriftliche Erlaubnis der Eltern notwendig. Wenn ein Verdacht auf eine CF durch das Screening gestellt wird, wird eines der acht pädiatrischen CFStudienzentren informiert, die die Eltern mit dem

Abbildung 1

Flow chart CF-Neugeborenen-Screening.

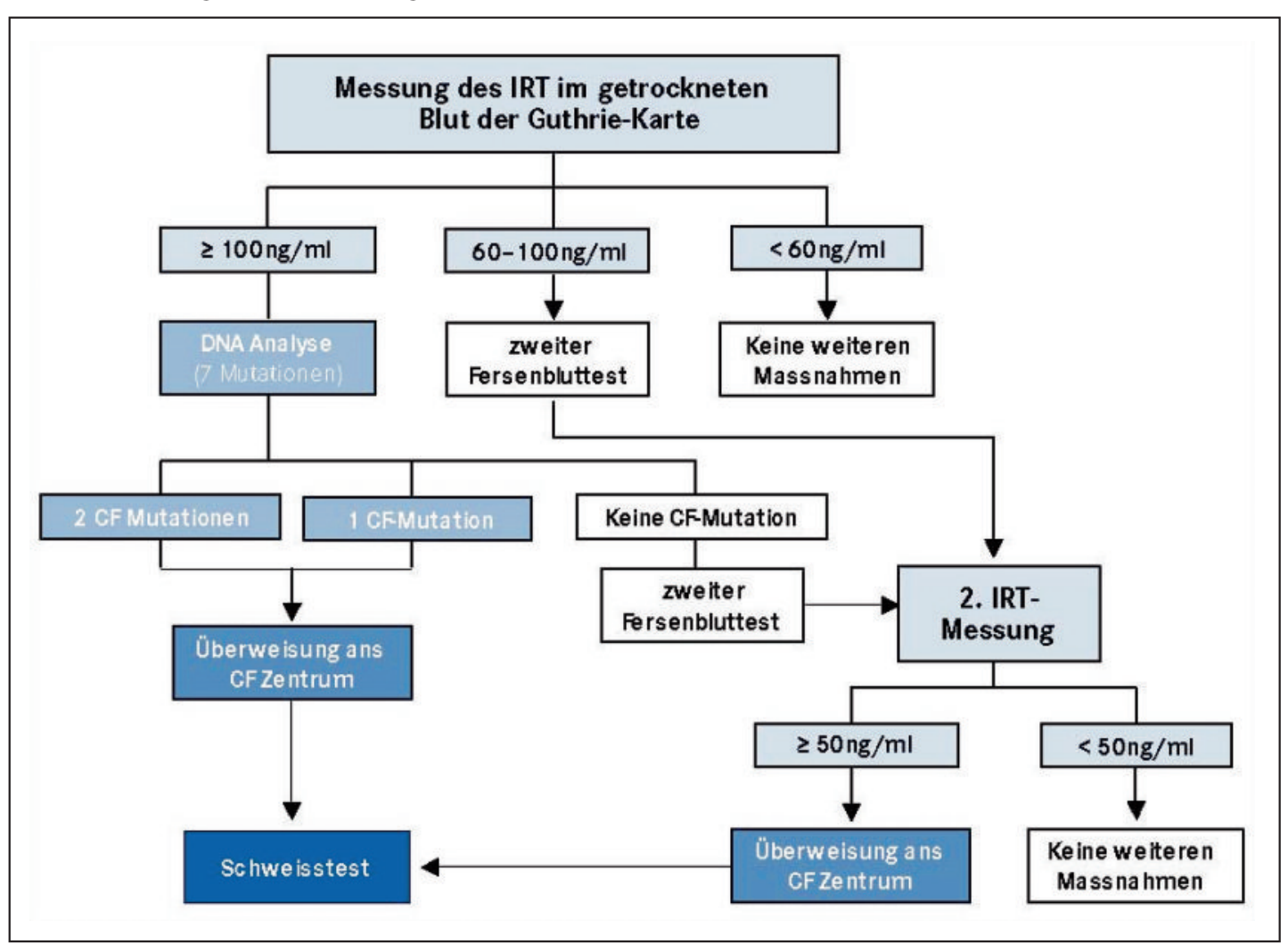


Kind zur weiteren Abklärung aufbieten. Bei negativer DNA-Analyse bzw. Borderline-Resultaten im IRT-Test wird - analog dem etablierten Neugeborenen-Screening - ein zweiter Guthrietest direkt vom NGS-Labor beim zuständigen Kinderarzt bzw. der zuständigen Hebamme eingefordert. Sollte bei diesem zweiten IRT-Test der Grenzwert von $50 \mathrm{ng} / \mathrm{ml}$ überschritten sein, wird ebenfalls ein CF-Zentrum zur weiteren Abklärung informiert (Abb.1). Im CF-Zentrum werden die weiteren diagnostischen Abklärungen gemäss den internationalen Richtlinien $[9,10]$ (Schweisstest, genetische Untersuchungen im Blut usw.) nur bei Zustimmung der Eltern gemacht und der Verdacht einer CF erhärtet oder verworfen. Ist der Schweisstest positiv, ist die Diagnose CF gesichert. Ist der Schweisstest negativ, werden keine weiteren Abklärungen ge-

\section{Literatur}

1 Balfour-Lynn IM. Newborn screening for cystic fibrosis: evidence for benefit. Arch Dis Child. 2008;93:7-10.

2 Crossley JR, Elliott RB, Smith PA. Dried-blood spot screening for cystic fibrosis in the newborn. Lancet. 1979;1:472-4.

3 Massie J, Clements B, Australian Paediatric Respiratory Group. Diagnosis of cystic fibrosis after newborn screening: the Australasian experience - twenty years and five million babies later: a consensus statement from the Australasian Paediatric Respiratory Group. Pediatr Pulmonol. 2005;39:440-6.

4 Grosse SD, Boyle CA, Botkin JR et al. Newborn screening for cystic fibrosis: Evaluation of benefits and risks and recommendations for state newborn screening programs. MMWR Recomm Rep 2004;53(RR-13):1-36.

\section{Die falschpositiven Resultate werden mit dem bei uns eingeführten zweistufigen Verfahren möglichst klein gehalten.}

macht. Diese Kinder können gesund sein und keine weiteren Erkrankungen haben, oder sie sind gesunde CF-Genträger, oder haben eine atypische $\mathrm{CF}$, die erst im Erwachsenenalter Symptome macht und meistens mild verläuft. Diese Eltern werden über die Möglichkeit des CF-Trägertums bzw. des Vorliegens einer milden atypischen CF-Variante aufgeklärt. Allen Eltern wird eine ausführliche genetische Beratung betreffend CF-Trägertum an einer der offiziellen genetischen Beratungsstellen in der Schweiz angeboten und sie erhalten ein Merkblatt dazu. Der betreuende Kinderarzt oder Hausarzt wird - im Einverständnis der Eltern dementsprechend informiert werden, damit er bei allfällig später auftretenden Symptomen auch adäquate Abklärungen machen kann.

Das jetzige Pilotprojekt ist vom Bundesamt für Gesundheit (BAG) für 2 Jahre bewilligt. Bei erfolgreicher Durchführung werden wir beim BAG frühzeitig eine reguläre Aufnahme der CF ins NGS beantragen, um eine kontinuierliche Fortsetzung $\mathrm{zu}$ gewährleisten.

Aufgrund der Bedeutung dieses Beitrags sowohl für Kinderund Jugendärztinnen und-ärzte als auch für ein breites ärztliches Publikum haben sich die Redaktionen der SÄZ und der Zeitschrift Paediatrica darauf geeinigt, den Text in beiden Zeitschriften zu publizieren. Die Referenz für Paediatrica lautet: Paediatrica 2010;21(5):38-39 (deutsche Version) [Paediatrica 2010;21(5):40-1 (französische Version)].
5 Southern KW, Munck A, Pollitt R et al. A survey of newborn screening for cystic fibrosis in Europe. J Cyst Fibros. 2007;6:57-65.

6 Comeau AM, White TB, Campbell PW 3rd et al Guidelines for implementation of cystic fibrosis newborn screening programs: Cystic Fibrosis Foundation workshop report. Pediatrics. 2007;119:e495-e518.

7 Castellani C, Southern KW, Brownlee K et al. European best practice guidelines for cystic fibrosis neonatal screening. J Cyst Fibros. 2010;8:153-73.

8 Brice P, Jarrett J, Mugford M. Genetic screening for cystic fibrosis: an overview of the science and the economics. J Cyst Fibros. 2007; 6:255-61.

9 Rosenstein BJ, Cutting GR, for the Cystic Fibrosis Foundation Consensus Panel. The diagnosis of cystic fibrosis: A consensus statement. J Pediatrics. 1998;132:589-95.

10 Farrell PM, Rosenstein BJ, White TB et al. Guidelines for diagnosis of cystic fibrosis in newborns through older adults: Cystic Fibrosis Foundation Consensus Report. J Pediatr. 2008; 153:S4-S14. 